ARKIV FÖR MATEMATIK Band 3 nr 18

Mitgeteilt am 9. Februar 1955 durch T. NAGELI und Otro Frostman

\title{
Zur Axiomatik endlicher Gruppen
}

\section{Teil}

\author{
Von Bengt Stolt
}

\section{\$ 1. Einleitung}

Die klassischen Definitionen einer Gruppe enthalten bekanntlich solche Axiome, die in weniger umfassende Axiome zerlegt werden können. In [1] habe ich eine solche Zerlegung vorgenommen. Dann habe ich Axiomensysteme gebildet, die für eine zugrundeliegende unendliche oder endliche Menge vollständig bzw. unvollständig sind; siehe [1], [2] und [3]. In späteren Arbeiten habe ich Systeme betrachtet, die nur für eine zugrundeliegende endliche Menge vollständig sind. Zunächst sind alle solche Systeme aufgestellt worden, die aus den sogenannten allgemeinen Axiomen, den Eins-Existenzaxiomen und den allgemeinen Inversaxiomen gebildet werden können, siehe [4]. Wenn die Komposition der Menge eindeutig ist, habe ich auch alle solchen Systeme aufgestellt, die nur für eine zugrundeliegende endliche Menge vollständig sind; siehe [5].

Es ist nun das Ziel der vorliegenden Arbeit, die übrigen Systeme zu bestimmen, die für eine zugrundeliegende endliche Menge vollständig sind. In der Arbeit werden 13 solche Systeme bestimmt; die Vollständigkeit zweier weiteren Systeme sind unentschieden. Für Bezeichnungen und Hilfssätze wird an [1] verwiesen.

\section{§ 2. Hilfssätze}

Zuerst wollen wir die folgenden Hilfssätze beweisen.

Hilfssatz 1. Wenn $A, E, l I(E)$ und $l i(U)$ bestehen, hat ein Element mit $l I(E)$ sogar die Eigenschaft lE.LI.

Beweis: Wenn $a$ ein beliebiges Element ist, ist es möglich, der Reihe nach die Produkte $a a \supset a^{2}, a a^{2} \supset a^{3}, \ldots$ zu bilden. Weil die zugrundeliegende Menge endlich ist, kommt man zu ciner Verknüpfung $a a^{n} \supset a^{m}, m \leqq n$. Dann ergibt sich

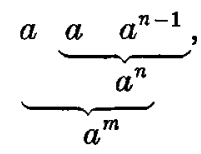

woraus $a a \supset a_{1}^{2}$ und $a_{1}^{2} a^{n-1} \supset a^{m}$ folgt. Ferner bilden wir 
B. SToLt, Zur Axiomatik endlicher Gruppen. II

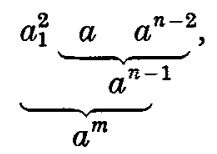

woraus $a_{1}^{2} a \supset a_{1}^{3}$ und $a_{1}^{3} a^{n-2} \supset a^{m}$ folgt. Indem man fortfährt, kornmt man schliesslich zu $a_{1}^{k-1} a \supset a_{1}^{k}$ und $a_{1}^{k} a^{m} \supset a^{m}$.

Wenn $e$ ein Element mit $\operatorname{lr}(E)$ ist, gilt auch $e a^{m} \supset a^{m}$. Aus $l i(U)$ folgt dann $a_{1}^{k}=e$. Wegen $a_{1}^{k-1} a \supset e$ gilt folglich $l E . l I$, womit der Hilfssatz bewiesen ist.

Hilfssatz 2. Wenn $A, E, l i(U)$ und $I(U)$ bestehen, gibt es ein Element mit $l E . I$ und $r E . I$.

Beweis: Wenn $a$ beliebig ist, ist es möglich, wie im vorigen Hilfssatz der Reihe nach die Produkte $a a \supset a^{2}, a a^{2} \supset a^{3}$ zu bilden, bis man zu $a a^{n} \supset a^{m}$, $m \leqq n$, kommt. Bilden wir ferner der Reihe nach $a a \supset a_{1}^{2}$ und $a_{1}^{2} a^{n-1} \supset a^{m}$, $a_{1}^{2} a \supset a_{1}^{3}$ und $a_{1}^{3} a^{n-2} \supset a^{m}$, kommen wir schliesslich zu den Ausdrücken $a_{1}^{k-1} a \supset a_{1}^{k}$ und $a_{1}^{k} a^{m} \supset a^{m}$. Wegen Hilfssatz 2 in [1], S. 35, besteht dann $a_{1}^{k} a_{1}^{k} \supset a_{1}^{k}$.

Wenn $b$ ein anderes Element ist, kann man dieselben Überlegungen durchführen. Man erhält dann $b_{1}^{h} b_{1}^{h} \supset b_{1}^{h}$. Wege $I(U)$ gilt nun

$$
a_{1}^{k}=b_{1}^{h}=\cdots=e_{\alpha},
$$

wo $e_{\alpha}$ die Eigenschaft $e_{\alpha} e_{\alpha} \supset e_{\alpha}$ hat. Wegen $a_{1}^{k-1} a \supset e_{\alpha}$ hat $e_{\alpha}$ sogar die Eigenschaft $l E . I$

Wir bilden nun

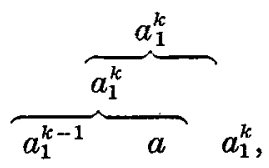

woraus $a a_{1}^{k} \supset a_{1}^{k+1}$ und $a_{1}^{k-1} a_{1}^{k+1} \supset a_{1}^{k}$ folgt. Indem man fortfährt, erhält man $a a_{1}^{k+1} \supset a_{1}^{k+2}$ und $a_{1}^{k-2} a_{1}^{k+2} \supset a_{1}^{k}, \ldots$, bis man zu $a a_{1}^{2 k-1} \supset a_{1}^{k}$ kommt. Folglich besteht auch rE.I, womit der Hilfssatz bewiesen ist.

\section{§ 3. Vollständige Systeme}

Wir wollen nun die Vollständigkeit der folgenden Systeme zeigen.
1) $A, E, l U, r I(U)$
2) $A, E, l U, r E . i$
3) $A, E, l U, r \varepsilon . i, r v . i$
4) $A, E, l E . l i, r U . l i, r i(U)$
5) $A, E, l E . r i, r U . r i, l i(U)$
6) $A, E, l U . l I, l i(U)$
7) $A, E, l U . r I, r i(U)$
8) $A, E, l U . l i, l i(U), I(U)$
9) $A, E, r U . l i, l i(U), I(U)$ 
10) $A, E, l U . r i, l \varepsilon . r i, l i(U), I(U)$

II) $A, E, l U . r i, r \varepsilon . r i, l i(U), I(U)$

12) $A, E, r U . r i, l \varepsilon . r i, l i(U), I(U)$

13) $A, E, r U . r i, r \varepsilon . r i, l i(U), I(U)$.

Vollständigkeitsbeweis von 1):

Wegen Hilfssatz 2 in [4] bestehen $A, E, l E, U, l U$ und $r I(U)$, womit 1) auf das vollständige System 12) in [1], S. 31, zurückgeführt ist.

Vollständigkeitsbeweis von 2):

Die Vollständigkeit von 2) ist in [4] bewiesen.

Vollständigkeitsbeweis von 3):

Wegen Hilfssatz 2 in [4] bestehen $A, E, l E, U, l U, r \varepsilon . i$ und $r v . i$, womit 1) auf das vollständige System 16) in [1], S. 32, zurückgeführt ist.

Vollständigkeitsbeweis von 4):

Wegen Hilfssatz 1 in [4] bestehen $A, E, l E, l i, r E . l i, l U . l i, r U . l i$ und $r i(U)$, womit 4) auf das vollständige System 18) in [1], S. 49, zurückgeführt, ist.

\section{Vollständigkeitsbeweis von 5):}

Wegen Hilfssatz 1 in [4] bestehen $A, E, l E . r i, r E . r i, l U . r i, r U . r i$ und $l i(U)$, womit 5) auf das vollständige System 17) in [1], S. 49, zurückgeführt ist.

\section{Vollständigkeitsbeweis von 6):}

Wegen Hilfssatz 1 hat ein Element mit $l U . l I$ auch die Eigenschaft $l E . l I$, womit 6) auf das vollständige System 1) in [1], S. 38, zurückgeführt ist.

\section{Vollständigkeitsbeweis von 7):}

Wegen Hilfssatz 1 hat ein Element mit $l U . r I$ auch die Eigenschaft $r E . r I$, womit 7) auf das vollständige System 10) in [1], S. 46, zurückgeführt ist.

\section{Vollständigkeitsbeweis von 8 ):}

Der Annahme zufolge gibt es ein Element $e$ mit $l U . l i$, das wegen Hilfssatz 2 in [1], S. 35, auch die Eigenschaft $l U . I$ hat. Wegen Hilfssatz 2 gibt es ein Element mit $l E . I$ und $r E . I$, and wegen $I(U)$ ist dieses Element gleich $e$. Weil also $e$ die Eigenschaften $l E . I$ und $l U . I$ hat, ist 8) auf das vollständige System 4) in $[1]$, S. 43, zurückgeführt.

\section{Vollständigkeitsbeweis von 9):}

Der Annahme zufolge gibt es ein Element $e$ mit $r U . l i$, das wegen Hilfssatz 2 in [1], S. 35, auch die Eigenschaft $l U . I$ hat. Wegen Hilfssatz 2 gibt es ein 


\section{B. stoLt, Zur Axiomatik endlicher Gruppen. II}

Element mit $l E . I$ und $r E . I$, und wegen $I(U)$ ist dieses Element gleich $e$. Weil also $e$ die Eigenschaften $l E . I$ und $r U . I$ hat, ist 9) auf das vollständige System 10) in [1], S. 46, zurückgeführt.

\section{Vollständigkeitsbeweis von 10):}

Der Annahme zufolge gibt es ein $e$, das $c e \supset c$ und $c^{\prime} c \supset e$ erfüllt. Aus

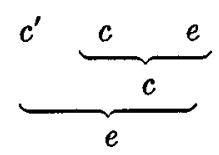

folgt $c^{\prime} c \supset e_{\alpha}$ und $e_{\alpha} e \supset e$. Wegen Hilfssatz 2 in [1], S. 35, gilt $e_{\alpha} e_{\alpha} \supset e_{\alpha}$, und wegen Hilfssatz 2 hat $e_{\alpha}$ die Eigenschaften $l E . I$ und $r E . I$. Wenn $a$ beliebig ist, gilt folglich $a a_{1}^{\prime} \supset e_{\alpha}$. Dann erhalten wir

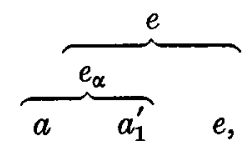

woraus $a_{1}^{\prime} e \supset a_{2}^{\prime}$ und $a a_{2}^{\prime} \supset e$ folgt. Also hat $e$ die Eigenschaft $r E . I$, womit 10) auf das vollständige System 6 a) in [3] zurückgeführt ist.

\section{Vollständigkeitsbeweis von 11):}

Der Annahme zufolge bestehen $c e \supset c$ und $c c_{1}^{\prime} \supset e$. Dann bilden wir

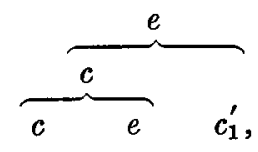

woraus $e c_{1}^{\prime} \supset c_{2}^{\prime}$ und $c c_{2}^{\prime} \supset e$ folgt,

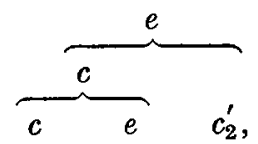

woraus $e c_{2}^{\prime} \supset c_{3}^{\prime}$ und $c c_{3}^{\prime} \supset e$ folgt, usw. Weil die zugrundeliegende Menge endlich ist, kommt man zu einer Verknüpfung $e c_{n}^{\prime} \supset c_{m}^{\prime}, m \leqq n$. Dann ergibt sich

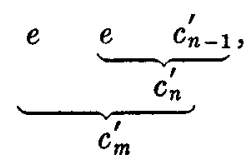

woraus $e e \supset e^{2}$ und $e^{2} c_{n-1}^{\prime} \supset c_{m}^{\prime}$ folgt, 
ARKIV FöR MATEMATIK. Bd $3 \mathrm{nr} 18$

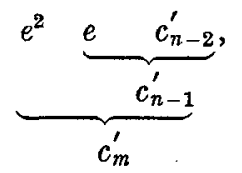

woraus $e^{2} e \supset e^{3}$ und $e^{3} \epsilon_{n-2}^{\prime} \supset c_{m}^{\prime}$ folgt, usw. Indem man fortfährt, erhält man schliesslich $e^{k} c_{m}^{\prime} \supset c_{m}^{\prime}$.

Wegen Hilfassatz 2 gibt es ein $e_{\alpha}$ mit $l E . I$ und $r E . I$. Wegen Hilfssatz 2 in [1], S. 35, und $I(U)$ gilt $e^{k}=e_{\alpha}$. Aus

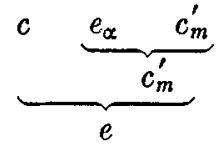

folgt $c e_{\alpha} \supset c$. Wegen $l E . I$ gilt $c_{\alpha_{1}}^{\prime} c \supset e_{\alpha}$. Dann ergibt sich

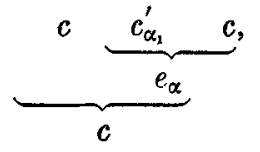

woraus $e_{c} c \supset c$ folgt. Wegen Hilfssatz 2 in [1], S. 35, und $I(U)$ gilt dann $e_{c}=e_{\alpha}$. Aus

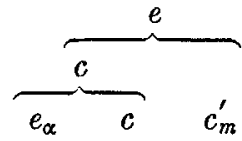

folgt $c c_{m}^{\prime} \supset e_{\beta}$ und $e_{\alpha} e_{\beta} \supset e$. Schliesslich bilden wir $a a_{1}^{\prime} \supset e_{\alpha}$, wo $a$ beliebig ist, und

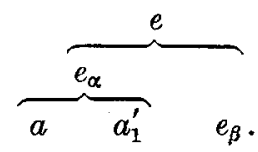

Dann gilt $a_{1}^{\prime} e_{\beta} \supset a_{2}^{\prime}$ und $a a_{2}^{\prime} \supset e . e$ hat also die Eigenschaften $r E . r i$ und $l U . r i$, womit 11) auf 4) zurückgeführt ist.

\section{Vollständigkeitsbeweis von 12):}

Auf Grund der Annahme bestehen $c e \supset c$ und $c_{1}^{\prime} c \supset e$, wo $e$ ein Element mit $r U . r i$ und $l_{\varepsilon . r i}$ ist.

Aus

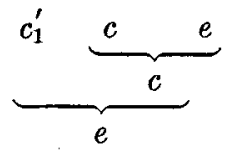

folgt $c_{1}^{\prime} c \supset e_{\beta}$ und $e_{\beta} e \supset e$. Wegen Hilfssatz 2 gibt es ein $e_{\alpha}$ mit $l E . I$ und $r E . I$, und wegen Hilfssatz 2 in [1], S. 35, und $I(U)$ gilt $e_{\beta}=e_{\alpha}$. 
B. Stolt, Zur Axiomatik endlicher Gruppen. II

Aus

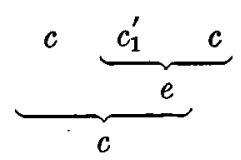

folgt $c c_{1}^{\prime} \supset e_{c}$ und $e_{c} c \supset c$. Wegen Hilfssatz 2 in [1], S. 35, und $I(U)$ gilt $e_{c}=e_{\alpha}$. Ferner bilden wir

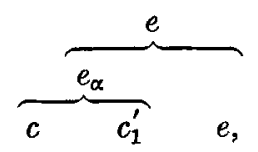

woraus $c_{1}^{\prime} e \supset c_{\alpha}^{\prime}$ und $c c_{\alpha}^{\prime} \supset e$ folgt, und

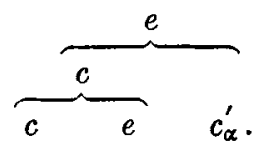

Dann folgt $e c_{\alpha}^{\prime} \supset c_{\alpha}^{\prime}$, und wegen Hilfssatz 2 in [1], S. 35, und $I(U)$ gilt ferner $e_{\alpha}=e$, womit 12) auf das vollständige System 17) in [1], S. 49, zurückgeführt ist.

Vollständigkeitsbeweis von 13):

Der Annahme zufolge bestehen $c e \supset c$ und $c c^{\prime} \supset e$, wo $e$ ein Element mit $r U . r i$ und $r \varepsilon . r i$ ist. Wegen Hilfssatz 2 gibt es ferner ein $e_{\alpha}$ mit $l E . I$ und $r E . I$. Aus

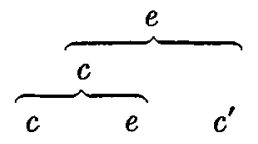

folgt $e c^{\prime} \supset c^{\prime}$. Wegen Hilfssatz 2 in $[1]$, S. 35, und $I(U)$ gilt dann $e_{\alpha}=e$, womit 13) auf das vollständige System 5) zurückgeführt ist.

\section{§ 4. Unentschiedene Systeme. Die Irreduzibilität der vollständigen Systeme}

Es ist dem Verfasser noch nicht gelungen, die Vollständigkeit oder Unvollständigkeit der folgenden zwei Systeme zu entscheiden. Es ist aber zu vermuten, dass wenigstens 1) unvollständig ist.

Die unentschiedenen Systeme sind wie folgt.

1) $A, E, l E, l U . r I, r \varepsilon . r i, r v . r i, l i(U), r I(U)$

2) $A, E, l U . r i, r U . r i, l i(U), I(U)$.

Zunächst werden wi zeigen, dass die Systeme 1)-4) und 6)-9) des $\$ 3$ irreduzibel sind. Zu diesem Zweck ersetzen wir die in jedem vollständigen System enthaltenen Axiome der Reihe nach mit weniger umfassenden Axiomen. 
ARKIV För MATEMATIK. Bd $3 \mathrm{nr} 18$

Es ist zu bemerken, dass in [1] gezeigt ist, dass ein vollständiges System das Axiom $A$, eines der Axiome $E, l E, r E$ und eines der Axiome $E, U$ enthalten muss.

Wenn die zugrundeliegende Menge unendlich ist, sind sämtliche Systeme des \$ 3 mit der Ausnahme von 5) unvollständig, was aus [1], Satz 1 und Satz 7 im Abschnitt IV, hervorgeht. Wenn die zugrundeliegende Menge von 5) unendlich ist, bekommt man ein System, das weniger umfassend als das unentschiedene System $7 \mathrm{~d}$ ) in [3] ist.

\begin{tabular}{|c|c|c|}
\hline Vollständiges System & $\begin{array}{c}\text { Teilsystem, das aus dem } \\
\text { vollständigen System } \\
\text { hervorgeht }\end{array}$ & $\begin{array}{l}\text { Auf Grund folgenden } \\
\text { Satzes in [5] ist das } \\
\text { Teilsystem unvollständig }\end{array}$ \\
\hline 1. $A, E, l U, r I(U)$ & $\begin{array}{l}A, E, l U . i, l i(U), r I(U) \\
A, E, l U\end{array}$ & $\begin{array}{ll}\text { Satz } & 8 \\
\text { Satz } & 1\end{array}$ \\
\hline 2. $A, E, l U, r E . i$ & $\begin{array}{l}A, E, l U . i, l i(U), r E . i \\
A, E, l U, r \varepsilon . i\end{array}$ & $\begin{array}{ll}\text { Satz } & 8 \\
\text { Satz } & 1\end{array}$ \\
\hline 3. $A, E, l U, r \varepsilon . i, r v . i$ & $\begin{array}{l}A, E, l U . i, l i(U), r \varepsilon . i, r v . i \\
A, E, l U, r v . i \\
A, E, l U, r \varepsilon . i\end{array}$ & $\begin{array}{ll}\text { Satz } & 8 \\
\text { Satz } & 2 \\
\text { Satz } & 1\end{array}$ \\
\hline 4. $A, E, l E . l i, r U . l i, r i(U)$ & $\begin{array}{l}A, E, l E . i, r U . i, r i(U) \\
A, E, l \varepsilon . l i, r U . l i, r i(U) \\
A, E, l E . l i, r v . l i, r i(U) \\
A, E, l E . l i, r U . l i\end{array}$ & $\begin{array}{ll}\text { Satz } & 8 \\
\text { Satz } & 9 \\
\text { Satz } & 8 \\
\text { Satz } & 7\end{array}$ \\
\hline 5. $A, E, l E . r i, r U . r i, l i(U)$ & $\begin{array}{l}A, E, l E . i, r U . i, l i(U) \\
A, E, l \varepsilon . r i, r U . r i, l i(U) \\
A, E, l E . r i, r v . r i, r I(U), l i(U) \\
A, E, l E . r i, r U . r i, l I(U)\end{array}$ & $\begin{array}{ll}\text { Satz } & 8 \\
\text { Satz } & 9 \\
\text { Satz } & 8 \\
\text { Satz } & 7\end{array}$ \\
\hline 6. $A, E, l U . l I, l i(U)$ & $\begin{array}{l}A, E, l U . I, l i(U) \\
A, E, l v . l I, l i(U) \\
A, E, l U . l I\end{array}$ & $\begin{array}{ll}\text { Satz } & 1 \\
\text { Satz } & 8 \\
\text { Satz } & 7\end{array}$ \\
\hline 7. $A, E, l U . r I, r i(U)$ & $\begin{array}{l}A, E, l U . I, r i(U) \\
A, E, l v \cdot r I, r i(U), l I(U) \\
A, E, l U . r I, r I(U)\end{array}$ & $\begin{array}{ll}\text { Satz } & 9 \\
\text { Satz } & 8 \\
\text { Satz } & 6\end{array}$ \\
\hline 8. $A, E, l U . l i, l i(U), I(U)$ & $\begin{array}{l}A, E, l U . i, l i(U), I(U) \\
A, E, l v . l i, l i(U), I(U) \\
A, E, l U . l i, I(U) \\
A, E, l U . l i, l i(U), r I(U)\end{array}$ & $\begin{array}{ll}\text { Satz } & 5 \\
\text { Satz } & 8 \\
\text { Satz } & 7 \\
\text { Satz } & 9\end{array}$ \\
\hline 9. $A, E, r U . l i, l i(U), I(U)$ & $\begin{array}{l}A, E, r U i, l i(U), I(U) \\
A, E, r v . l i, l i(U), I(U) \\
A, E, r U . l i, I(U) \\
A, E, r U . l i, l i(U), r I(U)\end{array}$ & $\begin{array}{ll}\text { Satz } & 5 \\
\text { Satz } & 8 \\
\text { Satz } & 7 \\
\text { Satz } & 9\end{array}$ \\
\hline
\end{tabular}


B. StoLt, Zur Axiomatik endlicher Gruppen. II

Fortsetzung

\begin{tabular}{|c|c|c|}
\hline Vollständiges System & $\begin{array}{c}\text { Teilsystem, das aus dem } \\
\text { vollständigen System } \\
\text { hervorgeht }\end{array}$ & $\begin{array}{l}\text { Auf Grund folgenden } \\
\text { Satzes in [5] ist das } \\
\text { Teilsystem unvollständig }\end{array}$ \\
\hline 10. $A, E, l U . r i, l \varepsilon . r i, l i(U), I(U)$ & $\begin{array}{l}A, E, l U . i, l \varepsilon . i, l i(U), I(U) \\
A, E, l v . r i, l \varepsilon . r i, l i(U), I(U) \\
A, E, l U . r i, l i(U), I(U) \\
A, E, l U . r i, l \varepsilon . r i, I(U) \\
A, E, l U . r i, l \varepsilon . r i, l i(U), r I(U)\end{array}$ & $\begin{array}{cc}\text { Satz } & 5 \\
\text { Satz } & 8 \\
- & \\
\text { Satz } & 7 \\
\text { Satz } & 9\end{array}$ \\
\hline 11. $A, E, l U . r i, r \varepsilon . r i, l i(U), I(U)$ & $\begin{array}{l}A, E, l U . i, r \varepsilon . i, l i(U), I(U) \\
A, E, l v . r i, r \varepsilon . r i, l i(U), I(U) \\
A, E, l U . r i, l i(U), I(U) \\
A, E, l U . r i, r \varepsilon . r i, I(U) \\
A, E, l U . r i, r \varepsilon . r i, l i(U), r I(U)\end{array}$ & $\begin{array}{r}\text { Satz } 5 \\
\text { Satz } 8 \\
- \\
\text { Satz } 7 \\
\text { Satz } 9\end{array}$ \\
\hline 12. $A, E, r U . r i, l \varepsilon . r i, l i(U), I(U)$ & $\begin{array}{l}A, E, r U . i, l \varepsilon . i, l i(U), I(U) \\
A, E, r v . r i, l \varepsilon . r i, l i(U), I(U) \\
A, E, r U . r i, l i(U), I(U) \\
A, E, r U . r i, l \varepsilon . r i, I(U) \\
A, E, r U . r i, l \varepsilon . r i, l i(U), r I(U)\end{array}$ & 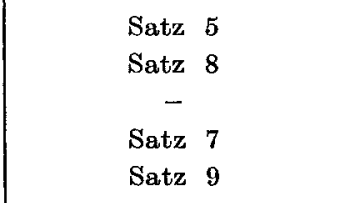 \\
\hline 13. $A, E, r U . r i, r \varepsilon . r i, l i(U), I(U)$ & $\begin{array}{l}A, E, r U . i, r \varepsilon . i, l i(U), I(U) \\
A, E, r v . r i, r \varepsilon . r i, l i(U), I(U) \\
A, E, r U . r i, \operatorname{li}(U), I(U) \\
A, E, r U . r i, r \varepsilon . r i, I(U) \\
A, E, r U . r i, r \varepsilon . r i, \operatorname{li}(U), r I(U)\end{array}$ & $\begin{array}{rr}\text { Satz } & 5 \\
\text { Satz } & 8 \\
- & \\
\text { Satz } & 7 \\
\text { Satz } & 9\end{array}$ \\
\hline
\end{tabular}

Aus den folgenden Tafeln ist es möglich zu bestimmen, ob ein gegebenes System vollständig, unvollständig oder unentschieden ist.

In den Tafeln sind folgende Bezeichnungen benutzt worden.

- bedeutet, dass das entsprechende System wegen eines Satzes des $\S 4$ in [5] unvollständig ist.

$G$ bedeutet, dass das entsprechende System mit einem System des $\S 3$ identisch ist. $g$ bedeutet, dass das entsprechende System für eine zugrundeliegende endliche aber nicht unendliche Menge vollständig ist.

$x$ bedeutet, dass das entsprechende System vollständig ist, wenn eine endliche oder unendliche Menge zugrunde liegt.

? bedeutet, dass das entsprechende System unentschieden ist.

Die Tafeln sind unter der Voraussetzung aufgestellt, dass das System $A, E$, $l E, r U . r i, l i(U)$ für eine zugrundeliegende unendliche Menge unvollständig ist. Wenn dieses System vollständig wäre, würde es notwendig sein, in einigen Fällen $g$ und $\boldsymbol{G}$ durch $x$ zu ersetzen. 
ARIIV FÖR MATEMATIK. Bd 3 nr 18

\begin{tabular}{|c|c|c|}
\hline Axiomensystem & $\begin{array}{c}\text { Auf Grund folgenden } \\
\text { vollständigen Systems } \\
\text { ist das Axiomensystem } \\
\text { vollständig }\end{array}$ & $\begin{array}{l}\text { Auf Grund folgenden } \\
\text { Satzes in [5] ist das } \\
\text { Axiomensystem } \\
\text { unvollständig }\end{array}$ \\
\hline$A, E, l E, r E, l U . e, r U . e . \ldots$ & & Satz 7 \\
\hline$A, E, l U, r E, i \ldots \ldots$ & 1 & \\
\hline$A, E, l U, r U . i \ldots \ldots$ & 2 & \\
\hline$A, E, l U, l I(E) \ldots \ldots . . . . . .$. & 6 & \\
\hline$A, E, l E, U, l U, r \varepsilon \cdot r I \ldots \ldots$ & & Satz 1 \\
\hline$A, E, l E, U, l U, r v . r I \ldots . . . .$. & & Satz 2 \\
\hline$A, E, l U, r \varepsilon . i, r v i \ldots \ldots$ & 3 & \\
\hline
\end{tabular}

\begin{tabular}{|c|c|c|c|c|c|c|c|c|c|c|c|c|}
\hline \multirow[b]{2}{*}{ Axiomensystem } & \multicolumn{12}{|c|}{ Eins-Unitätsaxiome, die zum Axiomensystem hinzugefügt werden sollen } \\
\hline & $\begin{array}{l}l I(U \\
r I(U\end{array}$ & ) $\begin{array}{l}r i(U \\
l I(U\end{array}$ & $\left\{\begin{array}{l}l i(U) \\
r I(U)\end{array}\right.$ & $r I(U)$ & $r i(U)$ & $l i(U)$ & $l I(U)$ & $I(U)$ & $\left.\right|_{\operatorname{li}(U)}(U)$ & $\mid l i(U)$ & $\mid \begin{array}{l}r i(U) \\
I(U)\end{array}$ & ) $\mid \begin{array}{ll}l i & (U) \\
r i & (U) \\
I & (U)\end{array}$ \\
\hline$A, E, l E, r E, l U . e, r U . e \ldots$. & - & $x$ & $x$ & - & $x$ & $x$ & - & - & $x$ & $x$ & $x$ & $x$ \\
\hline$A, E, l U . e, r U . e, l \varepsilon . e, r \varepsilon . e .$. & - & $g$ & $g$ & - & $g$ & $g$ & - & - & $g$ & $g$ & $g$ & $g$ \\
\hline$A, E, l U . e \ldots$ & - & $g$ & $g$ & - & $g$ & $g$ & - & - & $g$ & $g$ & $g$ & $g$ \\
\hline$A, E, l E, r E, l v . e, r v . e \ldots .$. & - & - & - & - & - & - & - & - & - & - & - & - \\
\hline$A, E, l E, r E, l U . l I, r U . l I$ & - & $x$ & $x$ & - & $x$ & $x$ & - & - & $x$ & $x$ & $x$ & $x$ \\
\hline$A, E, l E . l I, l U . l I . \ldots$. & - & $x$ & $x$ & -- & $x$ & $x$ & - & - & $x$ & $x$ & $x$ & $x$ \\
\hline$A, E, l E, l U . r I, r \varepsilon . r I$, rv.rI. & - & $x$ & $?$ & - & $x$ & $?$ & - & - & $x$ & $x$ & $x$ & $x$ \\
\hline$A, E, l E, l U . r I, r \varepsilon . r I \ldots$ & - & $x$ & $?$ & - & $x$ & - & - & - & $x$ & $x$ & $x$ & $x$ \\
\hline$A, E, l E, l U . r I, r v \cdot r I . .$. & - & $x$ & $?$ & - & $x$ & - & - & - & $x$ & $x$ & $x$ & $\boldsymbol{x}$ \\
\hline$A, E, l E . r I, l U . r I \ldots \ldots$ & - & $x$ & $?$ & - & $x$ & - & - & - & $x$ & $x$ & $x$ & $x$ \\
\hline$A, E, l U . l I, r U . l I, l \varepsilon . l I, r \varepsilon . l I .$. & - & - & $g$ & - & - & $g$ & - & - & $g$ & $g$ & $g$ & $g$ \\
\hline$A, E, l U . l I \ldots \ldots$ & - & - & $g$ & - & - & $G$ & - & - & $g$ & $g$ & $g$ & $g$ \\
\hline$A, E, r U . l I \ldots . .$. & - & - & $g$ & - & - & $\boldsymbol{G}$ & - & - & $g$ & $g$ & $g$ & $g$ \\
\hline$A, E, l E . I, r U . I \ldots \ldots$ & - & $x$ & $x$ & - & $x$ & $x$ & - & - & $x$ & $x$ & $x$ & $x$ \\
\hline$A, E, l E . I, l U . I \ldots \ldots$ & - & $x$ & - & - & $x$ & - & - & - & $x$ & $x$ & $x$ & $x$ \\
\hline$A, E, l E . I, l U . I, r \varepsilon . I$. & - & $x$ & - & - & $x$ & - & - & - & $x$ & $x$ & $x$ & $x$ \\
\hline$A, E, l E . I, l U . I, r v . I$ & - & $x$ & - & - & $x$ & - & - & - & $x$ & $x$ & $x$ & $x$ \\
\hline$A, E, l U . I, r U . I, r \varepsilon . I$, le.I $\ldots$ & - & - & - & - & - & - & - & - & - & $g$ & $g$ & $g$ \\
\hline$A, E, l U . I$. . . . . . & - & - & - & - & - & - & - & - & - & $g$ & $g$ & $g$ \\
\hline$A, E, l E . l i, r U . l i, r \varepsilon . l i \ldots$. & - & $x$ & $x$ & - & $x$ & $x$ & - & - & $x$ & $x$ & $x$ & $x$ \\
\hline$A, E, l E . r i, r U . r i, r \varepsilon . r i$. . & - & $x$ & $x$ & - & $x$ & $x$ & - & - & $x$ & $x$ & $x$ & $x$ \\
\hline$A, E, l E . l i, r U . l i, l v . l i \ldots$. & - & $x$ & $x$ & - & $x$ & $x$ & - & - & $x$ & $x$ & $x$ & $x$ \\
\hline$A, E, l E . r i, r U . r i, l v . r i$. . . & - & $x$ & $x$ & - & $x$ & $x$ & - & - & $x$ & $x$ & $x$ & $x$ \\
\hline$A, E, l E, r U . l i \ldots$ & - & $g$ & $x$ & - & $g$ & $x$ & - & - & $x$ & $x$ & $g$ & $x$ \\
\hline$A, E, l E, r U . r i \ldots .$. & - & $x$ & $g$ & - & $x$ & $g$ & - & - & $x$ & $x$ & $x$ & $x$ \\
\hline$A, E, l E, l U . l i \ldots$ & - & $x$ & $?$ & - & $x$ & - & - & - & $x$ & $x$ & $x$ & $x$ \\
\hline$A, E, l E, l U . r i \ldots$ & - & $x$ & $?$ & - & $\boldsymbol{x}$ & - & - & - & $x$ & $x$ & $x$ & $x$ \\
\hline$A, E, l E, l U . l i, r \varepsilon . l i \ldots .$. & - & $x$ & $?$ & - & $x$ & - & - & - & $x$ & $x$ & $x$ & $x$ \\
\hline$A, E, l E, l U . r i, r \varepsilon . r i \ldots .$. & - & $x$ & $?$ & - & $x$ & - & - & - & $x$ & $x$ & $x$ & $x$ \\
\hline$A, E, l E, l U . l i, r v . l i \ldots . .$. & - & $x$ & $?$ & - & $\boldsymbol{x}$ & - & - & - & $x$ & $x$ & $x$ & $x$ \\
\hline
\end{tabular}


B. sтоцт, Zur Axiomatik endlicher Gruppen. II

Fortsetzung

\begin{tabular}{|c|c|c|c|c|c|c|c|c|c|c|c|c|}
\hline \multirow[b]{2}{*}{ Axiomensystem } & \multicolumn{12}{|c|}{ Eins-Unitätsaxiome, die zum Axiomensystem hinzugefügt werden sollen } \\
\hline & $\begin{array}{l}l I(U) \\
r I(U)\end{array}$ & $\begin{array}{l}r i(U \\
l I(U\end{array}$ & $\left\{\begin{array}{l}l i(U) \\
r I(U)\end{array}\right.$ & $\gamma I(U)$ & $r i(U)$ & $l i(U)$ & $U I(U)$ & $I(U)$ & $\begin{array}{l}l i(U) \\
r i(U)\end{array}$ & $\left\{\begin{array}{l}l i(U) \\
I(U)\end{array}\right.$ & $\left\{\begin{array}{l}r i(U) \\
I(U)\end{array}\right.$ & ) $\mid \begin{array}{ll}l i & (U) \\
r i & (U) \\
I & (U)\end{array}$ \\
\hline$A, E, l E, l U . r i, r v \cdot r i \ldots .$. & - & $x$ & $?$ & - & $x$ & - & - & - & $\boldsymbol{x}$ & $x$ & $x$ & $x$ \\
\hline$A, E, l E . l i, l U . l i, r \varepsilon . l i, r v . l i$ & - & $x$ & $?$ & - & $x$ & $?$ & - & - & $x$ & $x$ & $x$ & $x$ \\
\hline$A, E, l E . r i, l U . r i, r \varepsilon . r i, r v . r i \ldots$ & - & $x$ & $?$ & - & $x$ & $?$ & - & - & $\boldsymbol{x}$ & $x$ & $x$ & $x$ \\
\hline$A, E, l E . l i, r U . l i . \ldots$ & - & $g$ & $x$ & - & $G$ & $x$ & - & - & $x$ & $x$ & $x$ & $x$ \\
\hline$A, E, l E . r i, r U . r i \ldots . . .$. & - & $x$ & $g$ & - & $x$ & $G$ & - & - & $x$ & $x$ & $x$ & $x$ \\
\hline$A, E, l E . l i, l U . l i \ldots$ & - & $x$ & - & - & $x$ & - & - & - & $x$ & $x$ & $x$ & $x$ \\
\hline$A, E, l E . r i, l U . r i \ldots . .$. & - & $x$ & - & - & $x$ & - & - & - & $x$ & $x$ & $x$ & $x$ \\
\hline$A, E, l U . l i \ldots \ldots$ & - & - & - & - & - & - & - & - & - & $\boldsymbol{G}$ & $?$ & $g$ \\
\hline$A, E, l U . r i \ldots . . . . . .$. & - & - & - & - & - & - & - & - & - & $?$ & $\boldsymbol{G}$ & $g$ \\
\hline$A, E, l U . l i, r U . l i$ & - & - & - & - & - & - & - & - & - & $g$ & $?$ & $g$ \\
\hline$A, E, l U . l i, l e . l i \ldots . .$. & - & - & - & - & - & - & - & - & - & $g$ & $\boldsymbol{G}$ & $g$ \\
\hline$A, E, l U . r i, l \varepsilon . r i$. . . . . . & - & - & - & - & - & - & - & - & - & $\boldsymbol{G}$ & $g$ & $g$ \\
\hline$A, E, l U . l i, r \varepsilon . l i$. . . & - & - & - & - & - & - & - & - & - & $g$ & $\boldsymbol{G}$ & $g$ \\
\hline$A, E, l U . r i, r \varepsilon . r i$. . . . & - & - & - & - & - & - & - & - & - & $\boldsymbol{G}$ & $g$ & $g$ \\
\hline$A, E, l E, r E, l U . i, r U . i .$. & - & - & - & - & - & - & - & - & - & - & - & - \\
\hline
\end{tabular}

\section{I T E R A T UR V E R Z E I CH N IS}

[1]. Stolt, B., Über Axiomensysteme, die eine abstrakte Gruppe bestimmen. Uppsala 1953.

[2]. - - Weitere Untersuchungen zur Gruppenaxiomatik. Ark. Mat. 3 Nr 5 (1954), 89-101.

[3]. - Über gewisse Axiomensysteme, die abstrakte Gruppen bestimmen. Ark. Mat. $3 \mathrm{Nr}$ 13 (1954), 187-191.

[4]. - Über irreduzible Axiomensysteme, die eine endliche abstrakte Gruppe bestimmen. Ark. Mat. $3 \mathrm{Nr} 7$ (1954) 113-115.

[5]. - Zur Axiomatik endlicher Gruppen. Ark. Mat. 3 Nr 11 (1954), 171-180.

Tryokt den 21 april 1955

Uppsala 1955. Almqvist \& Wiksells Boktryckeri AB 